\title{
Local Quasitriangular Hopf Algebras
}

Shouchuan ZHANG ${ }^{\dagger}$, Mark D. GOULD ${ }^{\ddagger}$ and Yao-Zhong ZHANG ${ }^{\ddagger}$

$\dagger$ Department of Mathematics, Hunan University, Changsha 410082, P.R. China

E-mail: z9491@yahoo.com.cn

$\ddagger$ Department of Mathematics, University of Queensland, Brisbane 4072, Australia

E-mail: mdg@maths.uq.edu.au,yzz@maths.uq.edu.au

Received January 31, 2008, in final form April 30, 2008; Published online May 09, 2008

Original article is available at http://www.emis.de/journals/SIGMA/2008/042/

\begin{abstract}
We find a new class of Hopf algebras, local quasitriangular Hopf algebras, which generalize quasitriangular Hopf algebras. Using these Hopf algebras, we obtain solutions of the Yang-Baxter equation in a systematic way. The category of modules with finite cycles over a local quasitriangular Hopf algebra is a braided tensor category.
\end{abstract}

Key words: Hopf algebra; braided category

2000 Mathematics Subject Classification: 16W30; 16G10

\section{Introduction}

The Yang-Baxter equation first came up in the paper by Yang as factorization condition of the scattering S-matrix in the many-body problem in one dimension and in the work by Baxter on exactly solvable models in statistical mechanics. It has been playing an important role in mathematics and physics (see $[2,16]$ ). Attempts to find solutions of the Yang-Baxter equation in a systematic way have led to the theory of quantum groups and quasitriangular Hopf algebras (see $[6,9])$.

Since the category of modules with finite cycles over a local quasitriangular Hopf algebra is a braided tensor category, we may also find solutions of the Yang-Baxter equation in a systematic way.

The main results in this paper are summarized in the following statement.

Theorem 1. (i) Assume that $\left(H,\left\{R_{n}\right\}\right)$ is a local quasitriangular Hopf algebra. Then $\left({ }_{H} \mathcal{M}^{\mathrm{cf}}\right.$, $\left.C^{\left\{R_{n}\right\}}\right),\left({ }_{H} \mathcal{M}^{\mathrm{dcf}}, C^{\left\{R_{n}\right\}}\right)$ and $\left({ }_{H} \mathcal{M}^{\mathrm{df}}, C^{\left\{R_{n}\right\}}\right)$ are braided tensor categories. Furthermore, if $\left(M, \alpha^{-}\right)$is an $H$-module with finite cycles and $R_{n+1}=R_{n}+W_{n}$ with $W_{n} \in H_{n+1} \otimes H_{(n+1)}$, then $\left(M, \alpha^{-}, \delta^{-}\right)$is a Yetter-Drinfeld $H$-module.

(ii) Assume that $B$ is a finite dimensional Hopf algebra and $M$ is a finite dimensional BHopf bimodule. Then $\left(\left(T_{B}(M)\right)^{\text {cop }} \bowtie_{\tau} T_{B^{*}}^{c}\left(M^{*}\right),\left\{R_{n}\right\}\right)$ is a local quasitriangular Hopf algebra. Furthermore, if $\left(k Q^{a}, k Q^{c}\right)$ and $\left(k Q^{s}, k Q^{s c}\right)$ are arrow dual pairings with finite Hopf quiver $Q$, then both $\left(\left(k Q^{a}\right)^{\mathrm{cop}} \bowtie_{\tau} k Q^{c},\left\{R_{n}\right\}\right)$ and $\left(\left(k Q^{s}\right)^{\mathrm{cop}} \bowtie_{\tau} k Q^{s c},\left\{R_{n}\right\}\right)$ are local quasitriangular Hopf algebras.

\section{Preliminaries}

Throughout, we work over a fixed field $k$. All algebras, coalgebras, Hopf algebras, and so on, are defined over $k$. Books $[7,11,15,13]$ provide the necessary background for Hopf algebras and book [1] provides a nice description of the path algebra approach.

Let $V$ and $W$ be two vector spaces. $\sigma_{V}$ denotes the map from $V$ to $V^{* *}$ by defining $\left\langle\sigma_{V}(x), f\right\rangle=\langle f, x\rangle$ for any $f \in V^{*}, x \in V . C_{V, W}$ denotes the map from $V \otimes W$ to $W \otimes V$ 
by defining $C_{V, W}(x \otimes y)=y \otimes x$ for any $x \in V, y \in W$. Denote $P$ by $\sum P^{\prime} \otimes P^{\prime \prime}$ for $P \in V \otimes W$. If $V$ is a finite-dimensional vector space over field $k$ with $V^{*}=\operatorname{Hom}_{k}(V, k)$. Define maps $b_{V}: k \rightarrow V \otimes V^{*}$ and $d_{V}: V^{*} \otimes V \rightarrow k$ by

$$
b_{V}(1)=\sum_{i} v_{i} \otimes v_{i}^{*} \quad \text { and } \quad \sum_{i, j} d_{V}\left(v_{i}^{*} \otimes v_{j}\right)=\left\langle v_{i}^{*}, v_{j}\right\rangle
$$

where $\left\{v_{i} \mid i=1,2, \ldots, n\right\}$ is any basis of $V$ and $\left\{v_{i}^{*} \mid i=1,2, \ldots, n\right\}$ is its dual basis in $V^{*} . d_{V}$ and $b_{V}$ are called evaluation and coevaluation of $V$, respectively. It is clear $\left(d_{V} \otimes i d_{U}\right)\left(i d_{U} \otimes b_{V}\right)=$ $i d_{U}$ and $\left(i d_{V} \otimes d_{V}\right)\left(b_{V} \otimes i d_{V}\right)=i d_{V} \cdot \xi_{V}$ denotes the linear isomorphism from $V$ to $V^{*}$ by sending $v_{i}$ to $v_{i}^{*}$ for $i=1,2, \ldots, n$. Note that we can define evaluation $d_{V}$ when $V$ is infinite.

We will use $\mu$ to denote the multiplication map of an algebra and use $\Delta$ to denote the comultiplication of a coalgebra. For a (left or right) module and a (left or right) comodule, denote by $\alpha^{-}, \alpha^{+}, \delta^{-}$and $\delta^{+}$the left module, right module, left comodule and right comodule structure maps, respectively. The Sweedler's sigma notations for coalgebras and comodules are $\Delta(x)=\sum x_{1} \otimes x_{2}, \delta^{-}(x)=\sum x_{(-1)} \otimes x_{(0)}, \delta^{+}(x)=\sum x_{(0)} \otimes x_{(1)}$. Let $(H, \mu, \eta, \Delta, \epsilon)$ be a bialgebra and let $\Delta^{\mathrm{cop}}:=C_{H, H} \Delta$ and $\mu^{\mathrm{op}}:=\mu C_{H, H}$. We denote $\left(H, \mu, \eta, \Delta^{\mathrm{cop}}, \epsilon\right)$ by $H^{\mathrm{cop}}$ and $\left(H, \mu^{\mathrm{op}}, \eta, \Delta, \epsilon\right)$ by $H^{\mathrm{op}}$. Sometimes, we also denote the unit element of $H$ by $1_{H}$.

Let $A$ and $H$ be two bialgebras with $\varnothing \neq X \subseteq A, \varnothing \neq Y \subseteq H$ and $P \in Y \otimes X, R \in Y \otimes Y$. Assume that $\tau$ is a linear map from $H \otimes A$ to $k$. We give the following notations.

$(Y, R)$ is called almost cocommutative if the following condition satisfied:

$$
(\mathrm{ACO}): \sum y_{2} R^{\prime} \otimes y_{1} R^{\prime \prime}=\sum R^{\prime} y_{1} \otimes R^{\prime \prime} y_{2} \quad \text { for any } \quad y \in Y .
$$

$\tau$ is called a skew pairing on $H \otimes A$ if for any $x, u \in H, y, z \in A$ the following conditions are satisfied:

$$
\begin{array}{ll}
(\mathrm{SP} 1): & \tau(x, y z)=\sum \tau\left(x_{1}, y\right) \tau\left(x_{2}, z\right) \\
(\mathrm{SP} 2): & \tau(x u, z)=\sum \tau\left(x, z_{2}\right) \tau\left(u, z_{1}\right) \\
(\mathrm{SP} 3): & \tau(x, \eta)=\epsilon_{H}(x) \\
(\mathrm{SP} 4): & \tau(\eta, y)=\epsilon_{A}(y)
\end{array}
$$

$P$ is called a copairing of $Y \otimes X$ if for any $x, u \in H, y, z \in A$ the following conditions are satisfied:

$$
\begin{aligned}
& (\mathrm{CP} 1): \sum P^{\prime} \otimes P_{1}^{\prime \prime} \otimes P_{2}^{\prime \prime}=\sum P^{\prime} Q^{\prime} \otimes Q^{\prime \prime} \otimes P^{\prime \prime} \quad \text { with } \quad P=Q ; \\
& (\mathrm{CP} 1): \sum P_{1}^{\prime} \otimes P_{2}^{\prime} \otimes P^{\prime \prime}=\sum P^{\prime} \otimes Q^{\prime} \otimes P^{\prime \prime} Q^{\prime \prime} \quad \text { with } \quad P=Q ; \\
& (\mathrm{CP} 3): \sum P^{\prime} \otimes \epsilon_{A}\left(P^{\prime \prime}\right)=\eta_{H} ; \\
& (\mathrm{CP} 4): \sum \epsilon_{H}\left(P^{\prime}\right) \otimes P^{\prime \prime}=\eta_{A} .
\end{aligned}
$$

For $R \in H \otimes H$ and two $H$-modules $U$ and $V$, define a linear map $C_{U, V}^{R}$ from $U \otimes V$ to $V \otimes U$ by sending $(x \otimes y)$ to $\sum R^{\prime \prime} y \otimes R^{\prime} x$ for any $x \in U, y \in V$.

If $V=\oplus_{i=0}^{\infty} V_{i}$ is a graded vector space, let $V_{>n}$ and $V_{\leq n}$ denote $\oplus_{i=n+1}^{\infty} V_{i}$ and $\oplus_{i=0}^{n} V_{i}$, respectively. We usually denote $\oplus_{i=0}^{n} V_{i}$ by $V_{(n)}$. If $\operatorname{dim} V_{i}<\infty$ for any natural number $i$, then $V$ is called a local finite graded vector space. We denote by $\iota_{i}$ the natural injection from $V_{i}$ to $V$ and by $\pi_{i}$ the corresponding projection from $V$ to $V_{i}$.

Let $H$ be a bialgebra and a graded coalgebra with an invertible element $R_{n}$ in $H_{(n)} \otimes H_{(n)}$ for any natural $n$. Assume $R_{n+1}=R_{n}+W_{n}$ with $W_{n} \in H_{(n+1)} \otimes H_{n+1}+H_{n+1} \otimes H_{(n+1)}$. $\left(H,\left\{R_{n}\right\}\right)$ is called a local quasitriangular bialgebra if $R_{n}$ is a copairing on $H_{(n)} \otimes H_{(n)}$, and 
$\left(H_{(n)}, R_{n}\right)$ is almost cocommutative for any natural number $n$. In this case, $\left\{R_{n}\right\}$ is called a local quasitriangular structure of $H$. Obviously, if $(H, R)$ is a quasitriangular bialgebra, then $\left(H,\left\{R_{n}\right\}\right)$ is a local quasitriangular bialgebra with $R_{0}=R, R_{i}=0, H_{0}=H, H_{i}=0$ for $i>0$.

The following facts are obvious: $\tau^{-1}=\tau\left(i d_{H} \otimes S\right)\left(\right.$ or $\left.=\tau\left(S^{-1} \otimes i d_{A}\right)\right)$ if $A$ is a Hopf algebra (or $H$ is a Hopf algebra with invertible antipode) and $\tau$ is a skew pairing; $P^{-1}=\left(S \otimes i d_{A}\right) P$ (or $=\left(i d_{H} \otimes S^{-1}\right) P$ ) if $H$ is a Hopf algebra (or $A$ is a Hopf algebra with invertible antipode) and $P$ is a copairing.

Let $A$ be an algebra and $M$ be an $A$-bimodule. Then the tensor algebra $T_{A}(M)$ of $M$ over $A$ is a graded algebra with $T_{A}(M)_{0}=A, T_{A}(M)_{1}=M$ and $T_{A}(M)_{n}=\otimes_{A}^{n} M$ for $n>1$. That is, $T_{A}(M)=A \oplus\left(\bigoplus_{n>0} \otimes_{A}^{n} M\right)$ (see [12]). Let $D$ be another algebra. If $h$ is an algebra map from $A$ to $D$ and $f$ is an $A$-bimodule map from $M$ to $D$, then by the universal property of $T_{A}(M)$ (see [12, Proposition 1.4.1]) there is a unique algebra map $T_{A}(h, f): T_{A}(M) \rightarrow D$ such that $T_{A}(h, f) \iota_{0}=h$ and $T_{A}(h, f) \iota_{1}=f$. One can easily see that $T_{A}(h, f)=h+\sum_{n>0} \mu^{n-1} T_{n}(f)$, where $T_{n}(f)$ is the map from $\otimes_{A}^{n} M$ to $\otimes_{A}^{n} D$ given by $T_{n}(f)\left(x_{1} \otimes x_{2} \otimes \cdots \otimes x_{n}\right)=f\left(x_{1}\right) \otimes f\left(x_{2}\right) \otimes \cdots \otimes f\left(x_{n}\right)$, i.e., $T_{n}(f)=f \otimes_{A} f \otimes_{A} \cdots \otimes_{A} f$. Note that $\mu$ can be viewed as a map from $D \otimes_{A} D$ to $D$. For the details, the reader is directed to [12, Section 1.4].

Dually, let $C$ be a coalgebra and let $M$ be a $C$-bicomodule. Then the cotensor coalgebra $T_{C}^{c}(M)$ of $M$ over $C$ is a graded coalgebra with $T_{C}^{c}(M)_{0}=C, T_{C}^{c}(M)_{1}=M$ and $T_{C}^{c}(M)_{n}=\square_{C}^{n} M$ for $n>1$. That is, $T_{C}^{c}(M)=C \oplus\left(\bigoplus_{n>0} \square_{C}^{n} M\right)$ (see [12]). Let $D$ be another coalgebra. If $h$ is a coalgebra map from $D$ to $C$ and $f$ is a $C$-bicomodule map from $D$ to $M$ such that $f(\operatorname{corad}(D))=0$, then by the universal property of $T_{C}^{c}(M)$ (see [12, Proposition 1.4.2]) there is a unique coalgebra map $T_{C}^{c}(h, f)$ from $D$ to $T_{C}^{c}(M)$ such that $\pi_{0} T_{C}^{c}(h, f)=h$ and $\pi_{1} T_{C}^{c}(h, f)=f$. It is not difficult to see that $T_{C}^{c}(h, f)=h+\sum_{n>0} T_{n}^{c}(f) \Delta_{n-1}$, where $T_{n}^{c}(f)$ is the map from $\square_{C}^{n} D$ to $\square_{C}^{n} M$ induced by $T_{n}(f)\left(x_{1} \otimes x_{2} \otimes \cdots \otimes x_{n}\right)=f\left(x_{1}\right) \otimes f\left(x_{2}\right) \otimes \cdots \otimes f\left(x_{n}\right)$, i.e., $T_{n}^{c}(f)=f \otimes f \otimes \cdots \otimes f$.

Furthermore, if $B$ is a Hopf algebra and $M$ is a $B$-Hopf bimodule, then $T_{B}(M)$ and $T_{B}^{c}(M)$ are two graded Hopf algebra. Indeed, by [12, Section 1.4] and [12, Proposition 1.5.1], $T_{B}(M)$ is a graded Hopf algebra with the counit $\varepsilon=\varepsilon_{B} \pi_{0}$ and the comultiplication $\Delta=\left(\iota_{0} \otimes \iota_{0}\right) \Delta_{B}+$ $\sum_{n>0} \mu^{n-1} T_{n}\left(\Delta_{M}\right)$, where $\Delta_{M}=\left(\iota_{0} \otimes \iota_{1}\right) \delta_{M}^{-}+\left(\iota_{1} \otimes \iota_{0}\right) \delta_{M}^{+}$. Dually, $T_{B}^{c}(M)$ is a graded Hopf algebra with multiplication $\mu=\mu_{B}\left(\pi_{0} \otimes \pi_{0}\right)+\sum_{n>0} T_{n}^{c}\left(\mu_{M}\right) \Delta_{n-1}$, where $\mu_{M}=\alpha_{M}^{-}\left(\pi_{0} \otimes \pi_{1}\right)+$ $\alpha_{M}^{+}\left(\pi_{1} \otimes \pi_{0}\right)$.

\section{Yang-Baxter equations}

Assume that $H$ is a bialgebra and a graded coalgebra with an invertible element $R_{n}$ in $H_{(n)} \otimes H_{(n)}$ for any natural $n$. For convenience, let (LQT1), (LQT2) and (LQT3) denote (CP1), (CP2) and $(\mathrm{ACO})$, respectively;

$$
\begin{array}{llll}
(\mathrm{LQT} 4): & R_{n+1}=R_{n}+W_{n} & \text { with } & W_{n} \in H_{(n+1)} \otimes H_{n+1}+H_{n+1} \otimes H_{(n+1)} \\
\left(\mathrm{LQT}^{\prime}\right): & R_{n+1}=R_{n}+W_{n} & \text { with } & W_{n} \in H_{n+1} \otimes H_{n+1} .
\end{array}
$$

Then $\left(H,\left\{R_{n}\right\}\right)$ is a local quasitriangular bialgebra if and only if (LQT1), (LQT2), (LQT3) and (LQT4) hold for any natural number $n$.

Let $H$ be a graded coalgebra and a bialgebra. A left $H$-module $M$ is called an $H$-module with finite cycles if, for any $x \in M$, there exists a natural number $n_{x}$ such that $H_{i} x=0$ when $i>n_{x}$. Let ${ }_{H} \mathcal{M}^{\text {cf }}$ denote the category of all left $H$-modules with finite cycles.

Lemma 1. Let $H$ be a graded coalgebra and a bialgebra. If $U$ and $V$ are left $H$-modules with finite cycles, so is $U \otimes V$. 
Proof. For any $x \in U, y \in V$, there exist two natural numbers $n_{x}$ and $n_{y}$, such that $H_{>n_{x}} x=0$ and $H_{>n_{y}} y=0$. Set $n_{x \otimes y}=2 n_{x}+2 n_{y}$. It is clear that $H_{>n_{x \otimes y}}(x \otimes y)=0$. Indeed, for any $h \in H_{i}$ with $i>n_{x \otimes y}$, we see

$$
h(x \otimes y)=\sum h_{1} x \otimes h_{2} y=0 \quad \text { (since } H \text { is graded coalgebra). }
$$

Lemma 2. Assume that $\left(H,\left\{R_{n}\right\}\right)$ is a local quasitriangular Hopf algebra. Then for any left $H$ modules $U$ and $V$ with finite cycles, there exists an invertible linear map $C_{U, V}^{\left\{R_{n}\right\}}: U \otimes V \rightarrow V \otimes U$ such that

$$
C_{U, V}^{\left\{R_{n}\right\}}(x \otimes y):=C^{R_{n}}(x \otimes y)=\sum R_{n}^{\prime \prime} y \otimes R_{n}^{\prime} x
$$

with $n>2 n_{x}+2 n_{y}$, for $x \in U, y \in V$.

Proof. We first define a map $f$ from $U \times V$ to $V \otimes U$ by sending $(x, y)$ to $\sum R_{n}^{\prime \prime} y \otimes R_{n}^{\prime} x$ with $n>2 n_{x}+2 n_{y}$ for any $x \in U, y \in V$. It is clear that $f$ is well defined. Indeed, if $n>2 n_{x}+2 n_{y}$, then $C^{R_{n+1}}(x \otimes y)=C^{R_{n}}(x \otimes y)$ since $R_{n+1}=R_{n}+W_{n}$ with $W_{n} \in H_{(n+1)} \otimes H_{n+1}+H_{n+1} \otimes H_{(n+1)}$. $f$ is a $k$-balanced function. Indeed, for $x, y \in U, z, w \in V, \alpha \in k$, let $n>2 n_{x}+2 n_{y}+2 n_{z}$. See

$$
\begin{aligned}
f(x+y, z) & =\sum R_{n}^{\prime \prime} z \otimes R_{n}^{\prime}(x+y)=\sum R_{n}^{\prime \prime} z \otimes R_{n}^{\prime} x+\sum R_{n}^{\prime \prime} z \otimes R_{n}^{\prime} y \\
& =f(x, z)+f(y, z) .
\end{aligned}
$$

Similarly, we can show that $f(x, z+w)=f(x, z)+f(x, w), f(x \alpha, z)=f(x, \alpha z)$. Consequently, there exists a linear map $C_{U, V}^{\left\{R_{n}\right\}}: U \otimes V \rightarrow V \otimes U$ such that

$$
C_{U, V}^{\left\{R_{n}\right\}}(x \otimes y)=C^{R_{n}}(x \otimes y)
$$

with $n>2 n_{x}+2 n_{y}$, for $x \in U, y \in V$.

The inverse $\left(C_{U, V}^{\left\{R_{n}\right\}}\right)^{-1}$ of $C_{U, V}^{\left\{R_{n}\right\}}$ is defined by sending $(y \otimes x)$ to $\sum\left(R_{n}^{-1}\right)^{\prime} x \otimes\left(R_{n}^{-1}\right)^{\prime \prime} y$ with $n>2 n_{x}+2 n_{y}$ for any $x \in U, y \in V$.

Theorem 2. Assume that $\left(H,\left\{R_{n}\right\}\right)$ is a local quasitriangular Hopf algebra. Then $\left(H \mathcal{M}^{\mathrm{cf}}\right.$, $\left.C^{\left\{R_{n}\right\}}\right)$ is a braided tensor category.

Proof. Since $H$ is a bialgebra, we have that $(H \mathcal{M}, \otimes, I, a, r, l)$ is a tensor category by [14, Proposition XI.3.1]. It follows from Lemma 1 that $\left(H \mathcal{M}^{\text {cf }}, \otimes, I, a, r, l\right)$ is a tensor subcategory of $\left({ }_{H} \mathcal{M}, \otimes, I, a, r, l\right) . C^{\left\{R_{n}\right\}}$ is a braiding of ${ }_{H} \mathcal{M}^{\mathrm{cf}}$, which can be shown by the way similar to the proof of [14, Proposition VIII.3.1, Proposition XIII.1.4].

An $H$-module $M$ is called a graded $H$-module if $M=\oplus_{i=0}^{\infty} M_{i}$ is a graded vector space and $H_{i} M_{j} \subseteq M_{i+j}$ for any natural number $i$ and $j$.

Lemma 3. Assume that $H$ is a local finite graded coalgebra and bialgebra. If $M=\oplus_{i=0}^{\infty} M_{i}$ is a graded $H$-module, then the following conditions are equivalent:

(i) $M$ is an $H$-module with finite cycles;

(ii) $H x$ is finite dimensional for any $x \in M$;

(iii) $H x$ is finite dimensional for any homogeneous element $x$ in $M$.

Proof. (i) $\Rightarrow$ (ii). For any $x \in M$, there exists a natural number $n_{x}$ such that $H_{i} x=0$ with $i>n_{x}$. Since $H /(0: x)_{H} \cong H x$, where $(0: x)_{H}:=\{h \in H \mid h \cdot x=0\}$, we have that $H x$ is finite dimensional.

(ii) $\Rightarrow$ (iii). It is clear. 
(iii) $\Rightarrow$ (i). We first show that, for any homogeneous element $x \in M_{i}$, there exists a natural number $n_{x}$ such that $H_{j} x=0$ with $j>n_{x}$. In fact, if the above does not hold, then there exists $h_{j} \in H_{n_{j}}$ such that $h_{j} x \neq 0$ with $n_{1}<n_{2}<\cdots$. Considering $h_{j} x \in M_{n_{j}+i}$ we have that $\left\{h_{j} x \mid j=1,2, \ldots\right\}$ is linear independent in $H x$, which contradicts to that $H x$ is finite dimensional.

For any $x \in M$, then $x=\sum_{i=1}^{l} x_{i}$ and $x_{i}$ is a homogeneous element for $i=1,2, \ldots, l$. There exists a natural number $n_{x_{i}}$ such that $H_{j} x_{i}=0$ with $j>n_{x_{i}}$. Set $n_{x}=\sum_{s=1}^{l} n_{x_{s}}$. Then $H_{j} x=0$ with $j>n_{x}$. Consequently, $M$ is an $H$-module with finite cycles.

Note that if $M=\oplus_{i=0}^{\infty} M_{i}$ is a graded $H$-module, then both (ii) $\Rightarrow$ (iii) and (iii) $\Rightarrow$ (i) hold in Lemma 3.

Let ${ }_{H} \mathcal{M}^{\text {gf }}$ and ${ }_{H} \mathcal{M}^{\text {gcf }}$ denote the category of all finite dimensional graded left $H$-modules and the category of all graded left $H$-modules with finite cycles. Obviously, they are two tensor subcategories of ${ }_{H} \mathcal{M}^{\mathrm{cf}}$. Therefore we have

Theorem 3. Assume that $\left(H,\left\{R_{n}\right\}\right)$ is a local quasitriangular Hopf algebra. Then $\left({ }_{H} \mathcal{M}^{\mathrm{gf}}\right.$, $\left.C^{\left\{R_{n}\right\}}\right)$ and $\left({ }_{H} \mathcal{M}^{\text {gcf }}, C^{\left\{R_{n}\right\}}\right)$ are two braided tensor categories.

Therefore, if $M$ is a finite dimensional graded $H$-module (or $H$-module with finite cycles) over local quasitriangular Hopf algebra $\left(H,\left\{R_{n}\right\}\right)$, then $C_{M, M}^{\left\{R_{n}\right\}}$ is a solution of Yang-Baxter equation on $M$.

It is easy to prove the following.

Theorem 4. Assume that $\left(H,\left\{R_{n}\right\}\right)$ is a local quasitriangular Hopf algebra and $R_{n+1}=R_{n}+W_{n}$ with $W_{n} \in H_{n+1} \otimes H_{(n+1)}$. If $\left(M, \alpha^{-}\right)$is an $H$-modules with finite cycles then $\left(M, \alpha^{-}, \delta^{-}\right)$is a Yetter-Drinfeld $H$-module, where $\delta^{-}(x)=\sum R_{n}^{\prime \prime} \otimes R_{n}^{\prime} x$ for any $x \in M$ and $n \geq n_{x}$.

\section{Relation between tensor algebras and co-tensor coalgebras}

Lemma 4 (See $[3,7]$ ). Let $A, B$ and $C$ be finite dimensional coalgebras, $\left(M, \delta_{M}^{-}, \delta_{M}^{+}\right)$and $\left(N, \delta_{N}^{-}, \delta_{N}^{+}\right)$be respectively a finite dimensional $A$-B-bicomodule and a finite dimensional $B-C$ bicomodule. Then

(i) $\left(M^{*}, \delta_{M}^{-*}, \delta_{M}^{+*}\right)$ is a finite dimensional $A^{*}-B^{*}$-bimodule;

(ii) $\left(M \square_{B} N, \delta_{M \square_{B} N}^{-}, \delta_{M \square_{B} N}^{+}\right)$is an A-C-bicomodule with structure maps $\delta_{M \square_{B} N}^{-}=\delta_{M}^{-} \otimes i d_{N}$ and $\delta_{M \square_{B} N}^{+}=i d_{M} \otimes \delta_{N}^{+}$;

(iii) $M^{*} \otimes_{B^{*}} N^{*} \cong\left(M \square_{B} N\right)^{*}$ (as $A^{*}$ - $C^{*}$-bimodules).

Lemma 5 (See $[3,7])$. Let $A, B$ and $C$ be finite dimensional algebras, $\left(M, \alpha_{M}^{-}, \alpha_{M}^{+}\right)$and $\left(N, \alpha_{N}^{-}, \alpha_{N}^{+}\right)$be respectively a finite dimensional $A-B$-bimodule and a finite dimensional $B-C$ bimodule. Then

(i) $\left(M^{*}, \alpha_{M}^{-*}, \alpha_{M}^{+*}\right)$ is a finite dimensional $A^{*}-B^{*}$-bicomodule;

(ii) $\left(M \otimes_{B} N, \alpha_{M \otimes_{B} N}^{-}, \alpha_{M \otimes_{B} N}^{+}\right)$is an A-C-bimodule with structure maps $\alpha_{M \otimes_{B} N}^{-}=\alpha_{M}^{-} \otimes i d_{N}$ and $\alpha_{M \otimes_{B} N}^{+}=i d_{M} \otimes \alpha_{N}^{+}$

(iii) $M^{*} \square_{B^{*}} N^{*} \cong\left(M \otimes_{B} N\right)^{*}$ (as $A^{*}$-C $C^{*}$-bicomodules).

Proof. (i) and (ii) are easy.

(iii) Consider

$$
\left.M^{*} \square_{B^{*}} N^{*} \cong\left(M^{*} \square_{B^{*}} N^{*}\right)^{* *} \cong\left(M \otimes_{B} N\right)^{*} \quad \text { (by Lemma } 4\right) .
$$

Of course, we can also prove it in the dual way of the proof of Lemma 4 , by sending $f \otimes_{k} g$ to $f \otimes_{B^{*}} g$ for any $f \in M^{*}, g \in N^{*}$ with $f \otimes g \in M^{*} \square_{B^{*}} N^{*}$. 
Theorem 5. If $A$ is a finite dimensional algebra and $M$ is a finite dimensional A-bimodule, then $T_{A}(M)$ is isomorphic to subalgebra $\sum_{n=0}^{\infty}\left(\square_{A^{*}}^{n} M^{*}\right)^{*}$ of $\left(T_{A^{*}}^{c}\left(M^{*}\right)\right)^{0}$ under map $\sigma_{T_{A}(M)}$ and $\sigma_{T_{A}(M)}=\sigma_{A}+\sum_{n>0} \mu^{n-1} T_{n}\left(\sigma_{M}\right)$ with $\mu^{n-1} T_{n}\left(\sigma_{M}\right)=\sigma_{\otimes_{A}^{n} M}$.

Proof. We view $\oplus_{n=0}^{\infty}\left(\square_{A^{*}}^{n} M^{*}\right)^{*}$ as inner direct sum of vector spaces. It is clear that $\sigma_{A}$ is algebra homomorphism from $A$ to $A^{* *} \subseteq\left(T_{A^{*}}^{c}\left(M^{*}\right)\right)^{*}$ and $\sigma_{M}$ is a $A$-bimodule homomorphism from $M$ to $M^{* *} \subseteq\left(T_{A^{*}}^{c}\left(M^{*}\right)\right)^{*}$. Thus it follows from [12, Proposition 1.4.1] that $\phi=\sigma_{A}+$ $\sum_{n>0} \mu^{n-1} T_{n}\left(\sigma_{M}\right)$ is an algebra homomorphism from $T_{A}(M)$ to $\left(T_{A^{*}}^{c}\left(M^{*}\right)\right)^{*}$.

It follows from Lemma 4 (iii) that $\mu^{n-1} T_{n}\left(\sigma_{M}\right)=\sigma_{\otimes_{A}^{n} M}$. Indeed, we use induction on $n>0$. Obviously, the conclusion holds when $n=1$. Let $n>1, N=\otimes_{A}^{n-1} M, L=\left(\square_{A^{*}}^{n-1} M^{*}\right)^{*}$ and $\zeta=\mu^{n-2} T_{n-1}\left(\sigma_{M}\right)$. Obviously, $\mu^{n-1} T_{n}\left(\sigma_{M}\right)=\mu\left(\zeta \otimes \sigma_{M}\right)$. By inductive assumption, $\zeta=\sigma_{N}$ is an $A$-bimodule isomorphism from $N$ to $L$. See

$$
\begin{aligned}
\otimes_{A}^{n} M=N \otimes_{A} M & \stackrel{\nu_{1}}{\cong} L \otimes_{A} M^{* *} \quad \text { (by inductive assumption) } \\
& \stackrel{\nu_{2}}{\cong}\left(\square_{A^{*}}^{n-1} M^{*}\right)^{*} \otimes_{A^{* *}} M^{* *} \\
& \left.\stackrel{\nu_{3}}{\cong}\left(\left(\square_{A^{*}}^{n-1} M^{*}\right) \square_{A^{*}} M^{*}\right)^{*} \quad \text { (by Lemma } 4 \text { (iii) }\right) \\
& =\left(\square_{A^{*}}^{n} M^{*}\right)^{*},
\end{aligned}
$$

where $\nu_{1}=\sigma_{N} \otimes_{A} \sigma_{M}, \nu_{2}\left(f^{* *} \otimes_{A} g^{* *}\right)=f^{* *} \otimes_{A^{* *}} g^{* *}$ and $\nu_{3}\left(f^{* *} \otimes_{A^{* *}} g^{* *}\right)=f^{* *} \otimes_{k} g^{* *}$ for any $f^{* *} \in\left(\square_{A^{*}}^{n-1} M^{*}\right)^{*}, g^{* *} \in M^{* *}$. Now we have to show $\nu_{3} \nu_{2} \nu_{1}=\sigma_{\otimes_{A}^{n} M}=\mu^{n-1} T_{n}\left(\sigma_{M}\right)$. For any $f^{*} \in \square_{A^{*}}^{n-1} M^{*}, g^{*} \in M^{*}, x \in \otimes_{A}^{n-1} M, y \in M$, on the one hand

$$
\left\langle\sigma_{\otimes_{A}^{n} M}\left(x \otimes_{A} y\right), f^{*} \otimes_{k} g^{*}\right\rangle=\left\langle f^{*}, x\right\rangle\left\langle g^{*}, y\right\rangle .
$$

On the other hand,

$$
\begin{aligned}
\left\langle\nu_{3} \nu_{2} \nu_{1}\left(x \otimes_{A} y\right), f^{*} \otimes_{k} g^{*}\right\rangle & =\left\langle\nu_{3} \nu_{2}\left(\sigma_{N}(x) \otimes_{A} \sigma_{M}(y)\right), f^{*} \otimes_{k} g^{*}\right\rangle \\
& =\left\langle\sigma_{M}(x) \otimes_{k} \sigma_{N}(y), f^{*} \otimes_{k} g^{*}\right\rangle=\left\langle f^{*}, x\right\rangle\left\langle g^{*}, y\right\rangle .
\end{aligned}
$$

Thus $\nu_{3} \nu_{2} \nu_{1}=\sigma_{\otimes_{A}^{n} M}$. See

$$
\begin{aligned}
\left\langle\mu\left(\zeta \otimes_{A} \sigma_{M}\right)\left(x \otimes_{A} y\right), f^{*} \otimes_{k} g^{*}\right\rangle & =\left\langle\zeta(x) \otimes_{A} \sigma_{M}(y), \Delta\left(f^{*} \otimes_{k} g^{*}\right)\right\rangle \\
& =\left\langle\zeta(x), f^{*}\right\rangle\left\langle\sigma_{M}(y), g^{*}\right\rangle=\left\langle f^{*}, x\right\rangle\left\langle g^{*}, y\right\rangle .
\end{aligned}
$$

Thus $\sigma_{\otimes_{A}^{n} M}=\mu^{n-1} T_{n}\left(\sigma_{M}\right)$.

Finally, for any $x \in T_{A}(M)$ with $x=x^{(1)}+x^{(2)}+\cdots+x^{(n)}$ and $x^{(i)} \in \otimes_{A}^{i} M$,

$$
\phi(x)=\sum_{i=1}^{n} \phi\left(x^{(i)}\right)=\sum_{i=1}^{n} \sigma_{\otimes_{A}^{i} M}\left(x^{(i)}\right)=\sigma_{T_{A}(M)}(x) .
$$

\section{5 (Co-)tensor Hopf algebras}

Lemma 6. Assume that $B$ is a finite dimensional Hopf algebra and $M$ is a finite dimensional $B$-Hopf bimodule. Let $A:=T_{B}(M)^{\mathrm{cop}}, H:=T_{B^{*}}^{c}\left(M^{*}\right)$. Then

(i) $\phi:=\sigma_{B}+\sum_{i>0} \mu^{i-1} T_{i}\left(\sigma_{M}\right)$ is a Hopf algebra isomorphism from $T_{B}(M)$ to the Hopf subalgebra $\sum_{i=0}^{\infty}\left(\square_{B^{*}}^{i} M^{*}\right)^{*}$ of $\left(T_{B^{*}}^{c}\left(M^{*}\right)\right)^{0}$;

(ii) Let $\phi_{n}:=\left.\phi\right|_{A_{(n)}}$ for any natural number $n \geq 0$. Then there exists $\psi_{n}:\left(H_{(n)}\right)^{*} \rightarrow A_{(n)}$ such that $\phi_{n} \psi_{n}=i d_{\left(H_{(n)}\right)^{*}}$ and $\psi_{n} \phi_{n}=i d_{A_{(n)}}$, and $\psi_{n+1}(x)=\psi_{n}(x)$ for any $x \in\left(H_{(n)}\right)^{*}$. Furthermore, $\phi_{n}$ and $\psi_{n}$ preserve the (co)multiplication operations of $T_{B}(M)$ and $\left(T_{B^{*}}^{c}\left(M^{*}\right)\right)^{0}$, respectively. 
Proof. (i) We first show that $\left(\square_{B^{*}}^{n} M^{*}\right)^{*} \subseteq\left(T_{B^{*}}^{c}\left(M^{*}\right)\right)^{0}$. For any $f \in\left(\square_{B^{*}}^{n} M^{*}\right)^{*}, \sum_{i=n+1}^{\infty} \square_{B^{*}}^{i} M^{*}$ $\subseteq \operatorname{ker} f$ and $\sum_{i=n+1}^{\infty} \square_{B^{*}}^{i} M^{*}$ is a finite codimensional ideal of $T_{B^{*}}^{c}\left(M^{*}\right)$. Consequently, $f \in$ $\left(T_{B^{*}}^{c}\left(M^{*}\right)\right)^{0}$.

Next we show that $\phi:=\sigma_{B}+\sum_{n>0} \mu^{n-1} T_{n}\left(\sigma_{M}\right)$ (see the proof of Theorem 5) is a coalgebra homomorphism from $T_{B}(M)$ to $\sum_{i=0}^{\infty}\left(\square_{B^{*}}^{c} M^{*}\right)^{*}$. For any $x \in \otimes_{B}^{n} M, f, g \in T_{B^{*}}^{c}\left(M^{*}\right)$, on the one hand

$$
\begin{aligned}
\langle\phi(x), f * g\rangle & =\langle f * g, x\rangle \quad \text { (by Theorem 5) } \\
& =\sum_{x}\left\langle f, x_{1}\right\rangle\left\langle g, x_{2}\right\rangle=\sum_{x}\left\langle\phi\left(x_{1}\right), f\right\rangle\left\langle\phi\left(x_{2}\right), g\right\rangle .
\end{aligned}
$$

On the other hand

$$
\langle\phi(x), f * g\rangle=\sum\left\langle(\phi(x))_{1}, f\right\rangle\left\langle(\phi(x))_{2}, g\right\rangle,
$$

since $\phi(x) \in\left(T_{B^{*}}^{c}\left(M^{*}\right)\right)^{0}$. Considering $T_{B^{*}}^{c}\left(M^{*}\right)=\oplus_{n \geq 0} \square_{B^{*}}^{n} M^{*} \cong \oplus_{n \geq 0}\left(\otimes_{B}^{n} M\right)^{*}$ as vector spaces, we have that $T_{B^{*}}\left(M^{*}\right)$ is dense in $\left(T_{B}(M)\right)^{*}$. Consequently, $\sum \phi\left(x_{1}\right) \otimes \phi\left(x_{2}\right)=$ $\sum(\phi(x))_{1} \otimes(\phi(x))_{2}$, i.e. $\phi$ is a coalgebra homomorphism.

(ii) It follows from Theorem 5 .

Recall the double cross product $A_{\alpha} \bowtie_{\beta} H$, defined in [17, p. 36]) and [14, Definition IX.2.2]. Assume that $H$ and $A$ are two bialgebras; $(A, \alpha)$ is a left $H$-module coalgebra and $(H, \beta)$ is a right $A$-module coalgebra. We define the multiplication $m_{D}$, unit $\eta_{D}$, comultiplication $\Delta_{D}$ and counit $\epsilon_{D}$ on $A \otimes H$ as follows:

$$
\begin{aligned}
& \mu_{D}((a \otimes h) \otimes(b \otimes g))=\sum a \alpha\left(h_{1}, b_{1}\right) \otimes \beta\left(h_{2}, b_{2}\right) g, \\
& \Delta_{D}(a \otimes h)=\sum\left(a_{1} \otimes h_{1} \otimes a_{2} \otimes h_{2}\right),
\end{aligned}
$$

$\epsilon_{D}=\epsilon_{A} \otimes \epsilon_{H}, \eta_{D}=\eta_{A} \otimes \eta_{H}$ for any $a, b \in A, h, g \in H$. We denote $\left(A \otimes H, \mu_{D}, \eta_{D}, \Delta_{D}, \epsilon_{D}\right)$ by $A_{\alpha} \bowtie_{\beta} H$, which is called the double cross product of $A$ and $H$.

Lemma 7 (See [8]). Let $H$ and $A$ be two bialgebras. Assume that $\tau$ is an invertible skew pairing on $H \otimes A$. If we define $\alpha(h, a)=\sum \tau\left(h_{1}, a_{1}\right) a_{2} \tau^{-1}\left(h_{2}, a_{3}\right)$ and $\beta(h, a)=\sum \tau\left(h_{1}, a_{1}\right) h_{2} \tau^{-1}\left(h_{3}, a_{2}\right)$ then the double cross product $A_{\alpha} \bowtie_{\beta} H$ of $A$ and $H$ is a bialgebra. Furthermore, if $A$ and $H$ are two Hopf algebras, then so is $A_{\alpha} \bowtie_{\beta} H$.

Proof. We can check that $(A, \alpha)$ is an $H$-module coalgebra and $(H, \beta)$ is an $A$-module coalgebra step by step. We can also check that (M1)-(M4) in [17, pp. 36-37] hold step by step. Consequently, it follows from [17, Corollary 1.8, Theorem 1.5] or [14, Theorem IX.2.3] that $A_{\alpha} \bowtie_{\beta} H$ is a Hopf algebra.

In this case, $A_{\alpha} \bowtie_{\beta} H$ can be written as $A \bowtie_{\tau} H$.

Lemma 8. Let $H$ and $A$ be two Hopf algebra. Assume that there exists a Hopf algebra monomorphism $\phi: A^{\mathrm{cop}} \rightarrow H^{0}$. Set $\tau=d_{H}\left(\phi \otimes i d_{H}\right) C_{H, A}$. Then $A \bowtie_{\tau} H$ is Hopf algebra.

Proof. Using [10, Proposition 2.4] or the definition of the evaluation and coevaluation on tensor product, we can obtain that $\tau$ is a skew pairing on $H \otimes A$. Considering Lemma 7 , we complete the proof.

Lemma 9. (i) If $H=\oplus_{n=0}^{\infty} H_{n}$ is a graded bialgebra and $H_{0}$ has an invertible antipode, then $H$ has an invertible antipode.

(ii) Assume that $B$ is a finite dimensional Hopf algebra and $M$ is a B-Hopf bimodule. Then both $T_{B}(M)$ and $T_{B}^{c}(M)$ have invertible antipodes. 
Proof. (i) It is clear that $H^{\mathrm{op}}$ is a graded bialgebra with $\left(H^{\mathrm{op}}\right)_{0}=\left(H_{0}\right)^{(\mathrm{op})}$. Thus $H^{\mathrm{op}}$ has an antipode by [12, Proposition 1.5.1]. However, the antipode of $H^{\mathrm{op}}$ is the inverse of antipode of $H$.

(ii) It follows from (i).

Lemma 10. Let $A=\oplus_{n=0}^{\infty} A_{n}$ and $H=\oplus_{n=0}^{\infty} H_{n}$ be two graded Hopf algebras with invertible antipodes. Let $\tau$ be a skew pairing on $(H \otimes A)$ and $P_{n}$ be a copairing of $H_{(n)} \otimes A_{(n)}$ for any natural number $n$. Set $D=A \bowtie_{\tau} H$ and $\left[P_{n}\right]=1_{A} \otimes P_{n} \otimes 1_{H}$. Then $\left(D,\left\{\left[P_{n}\right]\right\}\right)$ is almost cocommutative on $D_{(n)}$ if and only if

$$
\begin{aligned}
& (\mathrm{ACO} 1): \sum P^{\prime} y_{1} \otimes P^{\prime \prime} \otimes y_{2}=\sum y_{4} P^{\prime} \otimes P_{2}^{\prime \prime} \otimes y_{2} \tau\left(y_{1}, P_{1}^{\prime \prime}\right) \tau^{-1}\left(y_{3}, P_{3}^{\prime \prime}\right) \text { for any } y \in H_{(n)} ; \\
& (\mathrm{ACO} 2): \sum x_{2} \otimes P^{\prime} \otimes x_{1} P^{\prime \prime}=\sum x_{2} \otimes P_{2}^{\prime} \otimes P^{\prime \prime} x_{4} \tau\left(P_{1}^{\prime}, x_{1}\right) \tau^{-1}\left(P_{3}^{\prime}, x_{3}\right) \text { for any } x \in A_{(n)} .
\end{aligned}
$$

Proof. It is clear that $\left(D,\left\{\left[P_{n}\right]\right\}\right)$ is almost cocommutative on $D_{(n)}$ if and only if the following holds:

$$
\begin{aligned}
& \sum x_{2} \otimes y_{4} P^{\prime} \otimes x_{1} P_{2}^{\prime \prime} \otimes y_{2} \tau\left(y_{1}, P_{1}^{\prime \prime}\right) \tau^{-1}\left(y_{3}, P_{3}^{\prime \prime}\right) \\
& =\sum x_{2} \otimes P_{2}^{\prime} y_{1} \otimes P^{\prime \prime} x_{4} \otimes y_{2} \tau\left(P_{1}^{\prime}, x_{1}\right) \tau^{-1}\left(P_{3}^{\prime}, x_{3}\right)
\end{aligned}
$$

for any $x \in A_{(n)}, y \in H_{(n)}$.

Assume that both (ACO1) and (ACO2) hold. See that

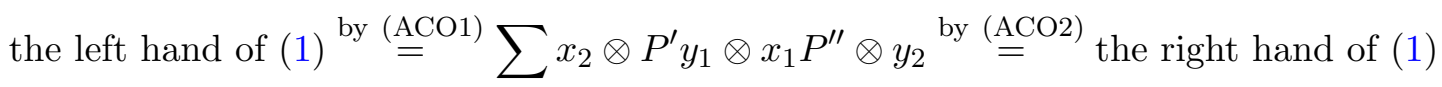

for any $x \in A_{(n)}, y \in H_{(n)}$. That is, (1) holds.

Conversely, assume that (1) holds. Thus we have that

$$
\begin{aligned}
& \sum x_{2} \otimes P^{\prime} \otimes x_{1} P_{2}^{\prime \prime} \otimes \epsilon_{H}\left(1_{H}\right) \tau\left(1_{H}, P_{1}^{\prime \prime}\right) \tau^{-1}\left(1_{H}, P_{3}^{\prime \prime}\right) \\
& =\sum x_{2} \otimes P_{2}^{\prime} \otimes P^{\prime \prime} x_{4} \otimes \epsilon_{H}\left(1_{H}\right) \tau\left(P_{1}^{\prime}, x_{1}\right) \tau^{-1}\left(P_{3}^{\prime}, x_{3}\right)
\end{aligned}
$$

and

$$
\begin{aligned}
& \sum \epsilon_{A}\left(1_{A}\right) \otimes y_{4} P^{\prime} \otimes P_{2}^{\prime \prime} \otimes y_{2} \tau\left(y_{1}, P_{1}^{\prime \prime}\right) \tau^{-1}\left(y_{3}, P_{3}^{\prime \prime}\right) \\
& =\sum \epsilon_{A}\left(1_{A}\right) \otimes P_{2}^{\prime} y_{1} \otimes P^{\prime \prime} \otimes y_{2} \tau\left(P_{1}^{\prime}, 1_{A}\right) \tau^{-1}\left(P_{3}^{\prime}, 1_{A}\right)
\end{aligned}
$$

for any $x \in A_{(n)}, y \in H_{(n)}$. Consequently, (ACO1) and (ACO2) hold.

Lemma 11. Let $A=\oplus_{n=0}^{\infty} A_{n}$ and $H=\oplus_{n=0}^{\infty} H_{n}$ be two graded Hopf algebras with invertible antipodes. Let $\tau$ be a skew pairing on $(H \otimes A)$ and $P_{n}$ be a copairing of $\left(H_{(n)} \otimes A_{(n)}\right)$ with $P_{n+1}=P_{n}+W_{n}$ and $W_{n} \in H_{(n+1)} \otimes A_{n+1}+H_{n+1} \otimes A_{(n+1)}$ for any natural number $n$. Set $D=A \bowtie_{\tau} H$. If $\tau\left(P_{n}^{\prime}, x\right) P_{n}^{\prime \prime}=x$ and $\tau\left(y, P_{n}^{\prime \prime}\right) P_{n}^{\prime}=y$ for any $x \in A_{(n)}, y \in H_{(n)}$, then $\left(D,\left\{\left[P_{n}\right]\right\}\right)$ is a local quasitriangular Hopf algebra.

Proof. It follows from Lemma 7 that $D=\oplus_{n=0}^{\infty} D_{n}$ is a Hopf algebra. Let $D_{n}=\sum_{i+j=n} A_{i} \otimes H_{j}$. It is clear that $D=\oplus_{n=0}^{\infty} D_{n}$ is a graded coalgebra. We only need to show that $\left(D,\left\{\left[P_{n}\right]\right\}\right)$ is almost cocommutative on $D_{(n)}$. Now fix $n$. For convenience, we denote $P_{n}$ by $P$ and $Q$ in the following formulae. For any $x \in A_{i}, y \in H_{j}$ with $i+j \leq n$,

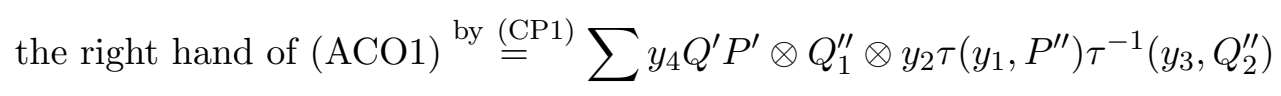




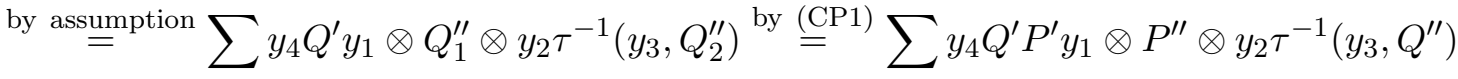

$$
\begin{aligned}
& =\sum y_{4} Q^{\prime} P^{\prime} y_{1} \otimes P^{\prime \prime} \otimes y_{2} \tau\left(S^{-1}\left(y_{3}\right), Q^{\prime \prime}\right) \stackrel{\text { by assumption }}{=} \sum y_{4} S^{-1}\left(y_{3}\right) P^{\prime} y_{1} \otimes P^{\prime \prime} \otimes y_{2} \\
& =\sum P^{\prime} y_{1} \otimes P^{\prime \prime} \otimes y_{2}=\text { the left hand of }(\mathrm{ACO} 1) \text {. }
\end{aligned}
$$

Similarly, we can show that $(\mathrm{ACO} 2)$ holds on $A_{(n)}$.

Theorem 6. Assume that $B$ is a finite dimensional Hopf algebra and $M$ is a finite dimenaional $B$-Hopf bimodule. Let $A:=T_{B}(M)^{\mathrm{cop}}, H:=T_{B^{*}}^{c}\left(M^{*}\right)$ and $D=A \bowtie_{\tau} H$ with $\tau:=d_{H}(\phi \otimes$ id) $C_{H, A}$. Then $\left(\left(T_{B}(M)\right)^{\mathrm{cop}} \bowtie_{\tau} T_{B^{*}}^{c}\left(M^{*}\right),\left\{R_{n}\right\}\right)$ is a local quasitriangular Hopf algebra. Here $P_{n}=\left(i d \otimes \psi_{n}\right) b_{H_{(n)}}, R_{n}=\left[P_{n}\right]=1_{B} \otimes\left(i d \otimes \psi_{n}\right) b_{H_{(n)}} \otimes 1_{B^{*}}, \phi$ and $\psi_{n}$ are defined in Lemma 6 .

Proof. By Lemma 9 (ii), $A$ and $H$ have invertible antipodes. Assume that $e_{1}^{(i)}, e_{2}^{(i)}, \ldots, e_{n_{i}}^{(i)}$ is a basis of $H_{i}$ and $e_{1}^{(i) *}, e_{2}^{(i) *}, \ldots, e_{n_{i}}^{(i) *}$ is an its dual basis in $\left(H_{i}\right)^{*}$. Then $\left\{e_{j}^{(i)} \mid i=0,1,2, \ldots, n\right.$; $\left.j=1,2, \ldots, n_{i}\right\}$ is a basis of $H_{(n)}$ and $\left\{e_{j}^{(i) *} \mid i=0,1,2, \ldots, n ; j=1,2, \ldots, n_{i}\right\}$ is its dual basis in $\left(H_{(n)}\right)^{*}$. Thus $b_{H_{(n)}}=\sum_{i=0}^{n} \sum_{j=1}^{n_{i}} e_{j}^{(i)} \otimes e_{j}^{(i) *}$. See that

$$
\begin{aligned}
P_{n+1} & =\sum_{i=0}^{n} \sum_{j=1}^{n_{i}} e_{j}^{(i)} \otimes \psi_{n+1}\left(e_{j}^{(i) *}\right)+\sum_{j=1}^{n_{n+1}} e_{j}^{(n+1)} \otimes \psi_{n+1}\left(e_{j}^{(n+1) *}\right) \\
& =P_{n}+\sum_{j=1}^{n_{n+1}} e_{j}^{(n+1)} \otimes \psi_{n+1}\left(e_{j}^{(n+1) *}\right) .
\end{aligned}
$$

Obviously, $\sum_{j=1}^{n_{n+1}} e_{j}^{(n+1)} \otimes \psi_{n+1}\left(e_{j}^{(n+1) *}\right) \in H_{n+1} \otimes A_{n+1}$. It is clear that $P_{n}$ is a copairing on $H_{(n)} \otimes A_{(n)}$ and $\tau$ is a skew pairing on $H \otimes A$ with $\sum \tau\left(P_{n}^{\prime}, x\right) P_{n}^{\prime \prime}=x$ and $\sum \tau\left(y, P_{n}^{\prime \prime}\right) P_{n}^{\prime}=y$ for any $x \in A_{(n)}, y \in H_{(n)}$. We complete the proof by Lemma 11 .

Note that $R_{n+1}=R_{n}+W_{n}$ with $W_{n} \in D_{n+1} \otimes D_{n+1}$ in the above theorem.

\section{Quiver Hopf algebras}

A quiver $Q=\left(Q_{0}, Q_{1}, s, t\right)$ is an oriented graph, where $Q_{0}$ and $Q_{1}$ are the sets of vertices and arrows, respectively; $s$ and $t$ are two maps from $Q_{1}$ to $Q_{0}$. For any arrow $a \in Q_{1}, s(a)$ and $t(a)$ are called its start vertex and end vertex, respectively, and $a$ is called an arrow from $s(a)$ to $t(a)$. For any $n \geq 0$, an $n$-path or a path of length $n$ in the quiver $Q$ is an ordered sequence of arrows $p=a_{n} a_{n-1} \cdots a_{1}$ with $t\left(a_{i}\right)=s\left(a_{i+1}\right)$ for all $1 \leq i \leq n-1$. Note that a 0 -path is exactly a vertex and a 1-path is exactly an arrow. In this case, we define $s(p)=s\left(a_{1}\right)$, the start vertex of $p$, and $t(p)=t\left(a_{n}\right)$, the end vertex of $p$. For a 0-path $x$, we have $s(x)=t(x)=x$. Let $Q_{n}$ be the set of $n$-paths, $Q_{(n)}$ be the set of $i$-paths with $i \leq n$ and $Q_{\infty}$ be the set of all paths in $Q$. Let ${ }^{y} Q_{n}^{x}$ denote the set of all $n$-paths from $x$ to $y, x, y \in Q_{0}$. That is, ${ }^{y} Q_{n}^{x}=\left\{p \in Q_{n} \mid s(p)=x, t(p)=y\right\}$. A quiver $Q$ is finite if $Q_{0}$ and $Q_{1}$ are finite sets.

Let $G$ be a group. Let $\mathcal{K}(G)$ denote the set of conjugate classes in $G . \quad r=\sum_{C \in \mathcal{K}(G)} r_{C} C$ is called a ramification (or ramification data) of $G$, if $r_{C}$ is the cardinal number of a set for any $C \in \mathcal{K}(G)$. We always assume that the cardinal number of the set $I_{C}(r)$ is $r_{C}$. Let $\mathcal{K}_{r}(G):=\left\{C \in \mathcal{K}(G) \mid r_{C} \neq 0\right\}=\left\{C \in \mathcal{K}(G) \mid I_{C}(r) \neq \varnothing\right\}$.

Let $G$ be a group. A quiver $Q$ is called a quiver of $G$ if $Q_{0}=G$ (i.e., $Q=\left(G, Q_{1}, s, t\right)$ ). If, in addition, there exists a ramification $r$ of $G$ such that the cardinal number of ${ }^{y} Q_{1}^{x}$ is equal to $r_{C}$ for any $x, y \in G$ with $x^{-1} y \in C \in \mathcal{K}(G)$, then $Q$ is called a Hopf quiver with respect to the ramification data $r$. In this case, there is a bijection from $I_{C}(r)$ to ${ }^{y} Q_{1}^{x}$. Denote by $(Q, G, r)$ the 
Hopf quiver of $G$ with respect to $r$. $e$ denotes the unit element of $G$. $\left\{p_{g} \mid g \in G\right\}$ denotes the dual basis of $\{g \mid g \in G\}$ of finite group algebra $k G$.

Let $Q=\left(G, Q_{1}, s, t\right)$ be a quiver of a group $G$. Then $k Q_{1}$ becomes a $k G$-bicomodule under the natural comodule structures:

$$
\delta^{-}(a)=t(a) \otimes a, \quad \delta^{+}(a)=a \otimes s(a), \quad a \in Q_{1},
$$

called an arrow comodule, written as $k Q_{1}^{c}$. In this case, the path coalgebra $k Q^{c}$ is exactly isomorphic to the cotensor coalgebra $T_{k G}^{c}\left(k Q_{1}^{c}\right)$ over $k G$ in a natural way (see [3] and [4]). We will regard $k Q^{c}=T_{k G}^{c}\left(k Q_{1}^{c}\right)$ in the following. Moreover, $k Q_{1}$ becomes a $(k G)^{*}$-bimodule with the module structures defined by

$$
p \cdot a:=\langle p, t(a)\rangle a, \quad a \cdot p:=\langle p, s(a)\rangle a, \quad p \in(k G)^{*}, \quad a \in Q_{1},
$$

written as $k Q_{1}^{a}$, called an arrow module. Therefore, we have a tensor algebra $T_{(k G)^{*}}\left(k Q_{1}^{a}\right)$. Note that the tensor algebra $T_{(k G)^{*}}\left(k Q_{1}^{a}\right)$ of $k Q_{1}^{a}$ over $(k G)^{*}$ is exactly isomorphic to the path algebra $k Q^{a}$. We will regard $k Q^{a}=T_{(k G)^{*}}\left(k Q_{1}^{a}\right)$ in the following.

Lemma 12 (See [4], Theorem 3.3, and [5], Theorem 3.1). Let $Q$ be a quiver over group $G$. Then the following statements are equivalent:

(i) $Q$ is a Hopf quiver.

(ii) Arrow comodule $k Q_{1}^{c}$ admits a $k G$-Hopf bimodule structure.

If $Q$ is finite, then the above statements are also equivalent to the following:

(iii) Arrow module $k Q_{1}^{a}$ admits a $(k G)^{*}$-Hopf bimodule structure.

Assume that $Q$ is a Hopf quiver. It follows from Lemma 12 that there exist a left $k G$ module structure $\alpha^{-}$and a right $k G$ - module structure $\alpha^{+}$on arrow comodule $\left(k Q_{1}^{c}, \delta^{-}, \delta^{+}\right)$ such that $\left(k Q_{1}^{c}, \alpha^{-}, \alpha^{+}, \delta^{-}, \delta^{+}\right)$becomes a $k G$-Hopf bimodule, called a co-arrow Hopf bimodule. We obtain two graded Hopf algebras $T_{k G}\left(k Q_{1}^{c}\right)$ and $T_{k G}^{c}\left(k Q_{1}^{c}\right)$, called semi-path Hopf algebra and co-path Hopf algebra, written as $k Q^{s}$ and $k Q^{c}$, respectively.

Assume that $Q$ is a finite Hopf quiver. Dually, it follows from Lemma 12 that there exist a left $(k G)^{*}$-comodule structure $\delta^{-}$and a right $(k G)^{*}$-comodule structure $\delta^{+}$on arrow module $\left(k Q_{1}^{a}, \alpha^{-}, \alpha^{+}\right)$such that $\left(k Q_{1}^{a}, \alpha^{-}, \alpha^{+}, \delta^{-}, \delta^{+}\right)$becomes a $(k G)^{*}$-Hopf bimodule, called an arrow Hopf bimodule. We obtain two graded Hopf algebras $T_{(k G)^{*}}\left(k Q_{1}^{a}\right)$ and $T_{(k G)^{*}}^{c}\left(k Q_{1}^{a}\right)$, called path Hopf algebra and semi-co-path Hopf algebra, written as $k Q^{a}$ and $k Q^{s c}$, respectively.

From now on, we assume that $Q$ is a finite Hopf quiver on finite group $G$. Let $\xi_{k Q_{1}^{a}}$ denote the linear map from $k Q_{1}^{a}$ to $\left(k Q_{1}^{c}\right)^{*}$ by sending $a$ to $a^{*}$ for any $a \in Q_{1}$ and $\xi_{k Q_{1}^{c}}$ denote the linear map from $k Q_{1}^{c}$ to $\left(k Q_{1}^{a}\right)^{*}$ by sending $a$ to $a^{*}$ for any $a \in Q_{1}$. It is easy to check the following.

Lemma 13. (i) If $\left(M, \alpha^{-}, \alpha^{+}, \delta^{-}, \delta^{+}\right)$is a finite dimensional $B$-Hopf bimodule and $B$ is a finite dimensional Hopf algebra, then $\left(M^{*}, \delta^{-*}, \delta^{+*}, \alpha^{-*}, \alpha^{+*}\right)$ is a $B^{*}$-Hopf bimodule.

(ii) If $\left(k Q_{1}^{c}, \alpha^{-}, \alpha^{+}, \delta^{-}, \delta^{+}\right)$is a co-arrow Hopf bimodule, then there exist unique left $(k G)^{*}$ comodule operation $\delta_{k Q_{1}^{a}}^{-}$and right $(k G)^{*}$-comodule operation $\delta_{k Q_{1}^{a}}^{+}$such that $\left(k Q_{1}^{a}, \alpha_{k Q_{1}^{a}}^{-}, \alpha_{k Q_{1}^{+}}^{+}\right.$, $\left.\delta_{k Q_{1}^{a}}^{-}, \delta_{k Q_{1}^{a}}^{+}\right)$becomes a $(k G)^{*}$-Hopf bimodule and $\xi_{k Q_{1}^{a}}$ becomes a $(k G)^{*}$-Hopf bimodule isomorphism from $\left(k Q_{1}^{a}, \alpha_{k Q_{1}^{a}}^{-}, \alpha_{k Q_{1}^{a}}^{+}, \delta_{k Q_{1}^{a}}^{-}, \delta_{k Q_{1}^{a}}^{+}\right)$to $\left(\left(k Q_{1}^{c}\right)^{*}, \delta^{-*}, \delta^{+*}, \alpha^{-*}, \alpha^{+*}\right)$.

(iii) If $\left(k Q_{1}^{a}, \alpha^{-}, \alpha^{+}, \delta^{-}, \delta^{+}\right)$is an arrow Hopf bimodule, then there exist unique left $k G-$ module operation $\alpha_{k Q_{1}^{c}}^{-}$and right $k G$-module $\alpha_{k Q_{1}^{c}}^{+}$such that $\left(k Q_{1}^{c}, \alpha_{k Q_{1}^{c}}^{-}, \alpha_{k Q_{1}^{+}}^{+}, \delta_{k Q_{1}^{c}}^{-}, \delta_{k Q_{1}^{c}}^{+}\right)$become a $k G$-Hopf bimodule and $\xi_{k Q_{1}^{c}}$ becomes a $k G$-Hopf bimodule isomorphism from $\left(k Q_{1}^{c}, \alpha_{k Q_{1}^{c}}^{-}, \alpha_{k Q_{1}^{c}}^{+}\right.$, $\left.\delta_{k Q_{1}^{c}}^{-}, \delta_{k Q_{1}^{c}}^{+}\right)$to $\left(\left(k Q_{1}^{a}\right)^{*}, \delta^{-*}, \delta^{+*}, \alpha^{-*}, \alpha^{+*}\right)$.

(iv) $\xi_{k Q_{1}^{a}}$ is a $(k G)^{*}$-Hopf bimodule isomorphism from $\left(k Q_{1}^{a}, \alpha_{k Q_{1}^{a}}^{-}, \alpha_{k Q_{1}^{+}}^{+}, \delta_{k Q_{1}^{a}}^{-}, \delta_{k Q_{1}^{a}}^{+}\right)$to $\left(\left(k Q_{1}^{c}\right)^{*}\right.$, $\left.\delta_{k Q_{1}^{c}}^{-}, \delta_{k Q_{1}^{c}}^{+}, \alpha_{k Q_{1}^{c}}^{-}, \alpha_{k Q_{1}^{c}}^{+}\right)$if and only if $\xi_{k Q_{1}^{c}}$ becomes a $k G$-Hopf bimodule isomorphism from

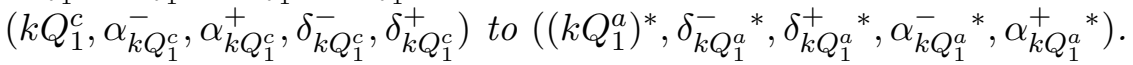


Let $B$ be a Hopf algebra and ${ }_{B}^{B} \mathcal{M}_{B}^{B}$ denote the category of $B$-Hopf bimodules. Let $G$ Hopf denote the category of graded Hopf algebras. Define $T_{B}(\psi)=: T_{B}\left(\iota_{0}, \iota_{1} \psi\right)$ and $T_{B}^{c}(\psi):=$ $T_{B}^{c}\left(\pi_{0}, \psi \pi_{1}\right)$ for any $B$-Hopf bimodule homomorphism $\psi$.

Lemma 14. Let $B$ be a Hopf algebra. Then $T_{B}$ and $T_{B}^{c}$ are two functors from ${ }_{B}^{B} \mathcal{M}_{B}^{B}$ to $G \mathcal{H}$ opf.

Proof. (i) If $\psi$ is a $B$-Hopf bimodule homomorphism from $M$ to $M^{\prime}$, then $T_{B}\left(\iota_{0}, \iota_{1} \psi\right)$ is a graded Hopf algebra homomorphism from $T_{B}(M)$ to $T_{B}\left(M^{\prime}\right)$. Indeed, let $\Phi:=T_{B}\left(\iota_{0}, \iota_{1} \psi\right)$. Then both $\Delta_{T_{B^{\prime}}\left(M^{\prime}\right)} \Phi$ and $(\Phi \otimes \Phi) \Delta_{T_{B}(M)}$ are graded algebra maps from $T_{B}(M)$ to $T_{B}\left(M^{\prime}\right) \otimes T_{B}\left(M^{\prime}\right)$. Now we show that $\Delta_{T_{B}\left(M^{\prime}\right)} \Phi=(\Phi \otimes \Phi) \Delta_{T_{B}(M)}$. Considering that $\Phi$ is an algebra homomorphism, we only have to show that $\Delta_{T_{B}\left(M^{\prime}\right)} \Phi \iota_{0}=(\Phi \otimes \Phi) \Delta_{T_{B}(M)} \iota_{0}$ and $\Delta_{T_{B}\left(M^{\prime}\right)} \Phi \iota_{1}=(\Phi \otimes \Phi) \Delta_{T_{B}(M)} \iota_{1}$. Obviously, the first equation holds. For the second equation, see

$$
\begin{aligned}
\Delta_{T_{B^{\prime}}\left(M^{\prime}\right)} \Phi \iota_{1} & =\Delta_{T_{B^{\prime}}\left(M^{\prime}\right)} \iota_{1} \psi=\left(\iota_{0} \otimes \iota_{1}\right) \delta_{M^{\prime}}^{-} \psi+\left(\iota_{1} \otimes \iota_{0}\right) \delta_{M^{\prime}}^{+} \psi \\
& =\left(\iota_{0} \otimes \iota_{1}\right)(i d \otimes \psi) \delta_{M}^{-}+\left(\iota_{1} \otimes \iota_{0}\right)(\psi \otimes i d) \delta_{M}^{+} \\
& =\left(\iota_{0} \otimes \iota_{1} \psi\right) \delta_{M}^{-}+\left(\iota_{1} \psi \otimes \iota_{0}\right) \delta_{M}^{+} \\
& =(\Phi \otimes \Phi)\left(\iota_{0} \otimes \iota_{1}\right) \delta_{M}^{-}+(\Phi \otimes \Phi)\left(\iota_{1} \otimes \iota_{0}\right) \delta_{M}^{+} \\
& =(\Phi \otimes \Phi)\left[\left(\iota_{0} \otimes \iota_{1}\right) \delta_{M}^{-}+\left(\iota_{1} \otimes \iota_{0}\right) \delta_{M}^{+}\right] \\
& =(\Phi \otimes \Phi) \Delta_{T_{B}(M)} \iota_{1} .
\end{aligned}
$$

Consequently, $T_{B}\left(\iota_{0}, \iota_{1} \psi\right)$ is a graded Hopf algebra homomorphisms.

(ii) If $\psi$ is a $B$-Hopf bimodule homomorphism from $M$ to $M^{\prime}$, then $T_{B}^{c}\left(\pi_{0}, \psi \pi_{1}\right)$ is a graded Hopf algebra homomorphism from $T_{B}^{c}(M)$ to $T_{B}^{c}\left(M^{\prime}\right)$. Indeed, let $\Psi:=T_{B}^{c}\left(\pi_{0}, \psi \pi_{1}\right)$. Then both $\Psi \mu_{T_{B}^{c}(M)}$ and $\mu_{T_{B}^{c}\left(M^{\prime}\right)}(\Psi \otimes \Psi)$ are graded coalgebra maps from $T_{B}^{c}(M) \otimes T_{B}^{c}(M)$ to $T_{B}^{c}\left(M^{\prime}\right)$. Since $T_{B}^{c}(M) \otimes T_{B}^{c}(M)$ is a graded coalgebra, $\operatorname{corad}\left(T_{B}^{c}(M) \otimes T_{B}^{c}(M)\right) \subseteq\left(T_{B}^{c}(M) \otimes T_{B}^{c}(M)\right)_{0}=$ $\iota_{0}(B) \otimes \iota_{0}(B)$. It follows that $\left(\pi_{1} \Psi \mu_{T_{B}^{c}(M)}\right)\left(\operatorname{corad}\left(T_{B}^{c}(M) \otimes T_{B}^{c}(M)\right)\right)=0$. Thus by the universal property of $T_{B}^{c}\left(M^{\prime}\right)$, in order to prove $\Psi \mu_{T_{B}^{c}(M)}=\mu_{T_{B}^{c}\left(M^{\prime}\right)}(\Psi \otimes \Psi)$, we only need to show $\pi_{n} \Psi \mu_{T_{B}^{c}(M)}=\pi_{n} \mu_{T_{B}^{c}\left(M^{\prime}\right)}(\Psi \otimes \Psi)$ for $n=0,1$. However, this follows from a straightforward computation dual to part (i). Furthermore, one can see $\Psi(1)=1$. Hence $\Psi$ is an algebra map, and so a Hopf algebra map.

(iii) It is straightforward to check $T_{B}(\psi) T_{B}\left(\psi^{\prime}\right)=T_{B}\left(\psi \psi^{\prime}\right)$ and $T_{B}^{c}(\psi) T_{B}^{c}\left(\psi^{\prime}\right)=T_{B}^{c}\left(\psi \psi^{\prime}\right)$ for $B$-Hopf bimodule homomorphisms $\psi: M^{\prime} \rightarrow M^{\prime \prime}$ and $\psi^{\prime}: M \rightarrow M^{\prime}$.

(iv) $T_{B}\left(i d_{M}\right)=i d_{T_{B}(M)}$ and $T_{B}^{c}\left(i d_{M}\right)=i d_{T_{B}^{c}(M)}$.

Lemma 15. If $\psi$ is a Hopf algebra isomorphism from $B$ to $B^{\prime}$ and $\left(M, \alpha^{-}, \alpha^{+}, \delta^{-}, \delta^{+}\right)$is a B-Hopf bimodule, then $\left(M, \alpha^{-}\left(\psi^{-1} \otimes i d_{M}\right), \alpha^{+}\left(i d_{M} \otimes \psi^{-1}\right),\left(\psi \otimes i d_{M}\right) \delta^{-},\left(i d_{M} \otimes \psi\right) \delta^{+}\right)$is a $B^{\prime}$-Hopf bimodule. Furthermore $T_{B}\left(\iota_{0} \psi, \iota_{1}\right)$ and $T_{B}^{c}\left(\psi \pi_{0}, \pi_{1}\right)$ are graded Hopf algebra isomorphisms from $T_{B}(M)$ to $T_{B^{\prime}}(M)$ and from $T_{B}^{c}(M)$ to $T_{B^{\prime}}^{c}(M)$, respectively.

By Lemma 14 and Lemma 13 (ii) and (iii), $T_{(k G)^{*}}\left(\iota_{0}, \iota_{1} \xi_{k Q_{1}^{a}}\right)$ and $T_{k G}^{c}\left(\pi_{0}, \xi_{k Q_{1}^{c}} \pi_{1}\right)$ are graded Hopf algebra isomorphisms from $T_{(k G)^{*}}\left(k Q_{1}^{a}\right)$ to $T_{(k G)^{*}}\left(\left(k Q_{1}^{c}\right)^{*}\right)$ and from $T_{k G}^{c}\left(k Q_{1}^{c}\right)$ to $T_{k G}^{c}\left(\left(k Q_{1}^{a}\right)^{*}\right)$, respectively. $T_{(k G)^{*}}^{c}\left(\pi_{0}, \xi_{k Q_{1}^{a}} \pi_{1}\right)$ and $T_{k G}\left(\iota_{0}, \iota_{1} \xi_{k Q_{1}^{c}}\right)$ are graded Hopf algebra isomorphisms from $T_{(k G)^{*}}^{c}\left(k Q_{1}^{a}\right)$ to $T_{(k G)^{*}}^{c}\left(\left(k Q_{1}^{c}\right)^{*}\right)$ and from $T_{k G}\left(k Q_{1}^{c}\right)$ to $T_{k G}\left(\left(k Q_{1}^{a}\right)^{*}\right)$, respectively. Furthermore, $\left(k Q_{1}^{a}, k Q_{1}^{c}\right),\left(k Q^{a}, k Q^{c}\right)$ and $\left(k Q^{s}, k Q^{s c}\right)$ are said to be arrow dual pairings.

Theorem 7. Assume that $(Q, G, r)$ is a finite Hopf quiver on finite group $G$. If $\left(k Q^{a}, k Q^{c}\right)$ and $\left(k Q^{s}, k Q^{s c}\right)$ are said to be arrow dual pairings, then

(i) $\left(\left(k Q^{a}\right)^{\mathrm{cop}} \bowtie_{\tau} k Q^{c},\left\{R_{n}\right\}\right)$ is a local quasitriangular Hopf algebra. Here

$$
R_{n}=\sum_{g \in G} p_{e} \otimes g \otimes p_{g} \otimes e+\sum_{q \in Q_{(n)}, q \notin G} p_{e} \otimes q \otimes q \otimes e
$$

and $\tau(a, b)=\delta_{a, b}$, for any two paths $a$ and $b$ in $Q$, where $\delta_{a, b}$ is the Kronecker symbol. 
(ii) There exist $\tau$ and $\left\{R_{n}\right\}$ such that $\left(\left(k Q^{s}\right)^{\mathrm{cop}} \bowtie_{\tau} k Q^{s c},\left\{R_{n}\right\}\right)$ becomes a local quasitriangular Hopf algebra.

Proof. (i) Let $B:=(k G)^{*}$ and $M:=k Q_{1}^{a}$. Thus $T_{B}(M)=k Q^{a}$. Since $\left(k Q_{1}^{a}, k Q_{1}^{c}\right)$ is an arrow dual pairing, $\xi_{k Q_{1}^{c}}$ is a $k G$-Hopf bimodule isomorphism from $k Q_{1}^{c}$ to $\left(k Q_{1}^{a}\right)^{*}$ by Lemma 13 . See that

$$
\begin{aligned}
T_{B^{*}}^{c}\left(M^{*}\right) & =T_{(k G)^{* *}}^{c}\left(\left(k Q_{1}^{a}\right)^{*}\right) \\
& \stackrel{\nu_{1}}{\cong} T_{k G}^{c}\left(\left(k Q_{1}^{a}\right)^{*}\right) \quad(\text { by Lemma 15) } \\
& \stackrel{\nu_{2}}{\cong} T_{k G}^{c}\left(k Q_{1}^{c}\right) \quad(\text { by Lemma } 13 \text { and Lemma 14) } \\
& =k Q^{c},
\end{aligned}
$$

where $\nu_{1}=T_{k G}^{c}\left(\sigma_{k G}^{-1} \pi_{0}, \pi_{1}\right), \nu_{2}=T_{(k G)^{* *}}^{c}\left(\pi_{0},\left(\xi_{k Q_{1}^{c}}\right)^{-1} \pi_{1}\right)$.

Let $H=T_{B^{*}}^{c}\left(M^{*}\right)$ and $A=T_{B}(M)^{\text {cop }}$. By Theorem $6,\left(\left(k Q^{a}\right)^{\text {cop }} \bowtie_{\tau} k Q^{c},\left\{R_{n}\right\}\right)$ is a local quasitriangular Hopf algebra. Here $\tau=d_{H}(\phi \otimes i d) C_{H, A}\left(\left(\nu_{2} \nu_{1}\right)^{-1} \otimes i d_{A}\right) ; R_{n}=\left(i d_{A} \otimes \nu_{2} \nu_{1} \otimes\right.$ $\left.i d_{A} \otimes \nu_{2} \nu_{1}\right)\left(1_{B} \otimes\left(i d \otimes \psi_{n}\right) b_{H_{(n)}} \otimes 1_{B^{*}}\right), \phi$ and $\psi_{n}$ are defined in Lemma 6 . We have to show that they are the same as in this theorem. That is,

$$
\begin{aligned}
& d_{H}(\phi \otimes i d) C_{H, A}\left(\left(\nu_{2} \nu_{1}\right)^{-1} \otimes i d_{A}\right)(a \otimes b)=\delta_{a, b} \\
&\left(1_{B} \otimes b_{H_{(n)}} \otimes 1_{B^{*}}\right)=\left(i d_{A} \otimes\left(\nu_{2} \nu_{1}\right)^{-1} \otimes \phi_{n} \otimes\left(\nu_{2} \nu_{1}\right)^{-1}\right) \\
& \times\left(\sum_{g \in G} p_{e} \otimes g \otimes p_{g} \otimes e+\sum_{q \in Q_{(n)}, q \notin G} p_{e} \otimes q \otimes q \otimes e\right) .
\end{aligned}
$$

If $b=b_{n} b_{n-1} \cdots b_{1}$ is a $n$-path in $k Q^{c}$ with $b_{i} \in Q_{1}$ for $i=1,2, \ldots, n$, then

$$
\left(\nu_{2} \nu_{1}\right)^{-1}(b)=b_{n}^{*} \otimes b_{n-1}^{*} \otimes \cdots \otimes b_{1}^{*} .
$$

If $b \in G$, then $\left(\nu_{2} \nu_{1}\right)^{-1}(b)=\sigma_{k G}(b)$. Consequently, for any $a, b \in Q_{\infty}$ with $b \in k Q^{c}$ and $a \in k Q^{a}$, we have

$$
\begin{aligned}
& d_{H}(\phi \otimes i d) C_{H, A}\left(\left(\nu_{2} \nu_{1}\right)^{-1} \otimes i d_{A}\right)(a \otimes b)=d_{H}\left(\phi(a) \otimes\left(\nu_{2} \nu_{1}\right)^{-1}(b)\right) \\
& = \begin{cases}\left\langle\phi(a), \sigma_{k G}(b)\right\rangle=\left\langle\sigma_{k G}(b), a\right\rangle=\delta_{a, b}, & \text { when } b \in Q_{0}, \\
\left\langle\left(\nu_{2} \nu_{1}\right)^{-1}, a\right\rangle \stackrel{\text { by }{ }^{\prime}(6)}{=} \delta_{a, b}, & \text { when } b \notin Q_{0} .\end{cases}
\end{aligned}
$$

Thus (4) holds. Note that $Q_{n}$ is a basis of not only $\left(k Q^{a}\right)_{n}$ but also $\left(k Q^{c}\right)_{n}$ for $n>0$. By (6), $\left\{\phi(q) \mid q \in Q_{n}\right\}$ is the dual basis of $\left\{\left(\nu_{2} \nu_{1}\right)^{-1}(q) \mid q \in Q_{n}\right\}$ for any $n>0$. Consequently, (5) holds.

(ii) Let $B:=k G$ and $M:=k Q_{1}^{c}$. Thus $T_{B}(M)=k Q^{s}$. Since $\left(k Q_{1}^{a}, k Q_{1}^{c}\right)$ is an arrow dual pairing, $\xi_{k Q_{1}^{a}}$ is a $(k G)^{*}$-Hopf bimodule isomorphism from $k Q_{1}^{a}$ to $\left(k Q_{1}^{c}\right)^{*}$ by Lemma 13. Thus

$$
\begin{aligned}
T_{B^{*}}^{c}\left(M^{*}\right) & =T_{(k G)^{*}}^{c}\left(\left(k Q_{1}^{c}\right)^{*}\right) \\
& \stackrel{\nu_{3}}{\cong} T_{(k G)^{*}}^{c}\left(k Q_{1}^{a}\right) \quad(\text { by Lemma } 13 \text { and Lemma 14) } \\
& =k Q^{s c}
\end{aligned}
$$

where $\nu_{3}=T_{(k G)^{*}}^{c}\left(\pi_{0},\left(\xi_{k Q_{1}^{a}}\right)^{-1} \pi_{1}\right)$. By Theorem 6 , the double cross product of $T_{B}(M)^{\text {cop }}$ and $T_{B^{*}}\left(M^{*}\right)$ is a local quasitriangular Hopf algebra. Consequently, so is the double cross product of $\left(k Q^{s}\right)^{\text {cop }}$ and $k Q^{s c}$. 
Note (LQT4') holds in the above theorem.

Example 1. Let $G=\mathbf{Z}_{2}=(g)=\{e, g\}$ be the group of order 2 with char $k \neq 2, X$ and $Y$ be respectively the set of arrows from $g^{0}$ to $g^{0}$ and the set of arrows from $g$ to $g$, and $|X|=|Y|=3$. The quiver $Q$ is a Hopf quiver with respect to ramification $r=r_{\{e\}}\{e\}$ with $r_{\{e\}}=3$. Let $\chi_{e}^{(i)} \in$ $\mathbf{Z}_{2}$ and $a_{y, x}^{(i)}$ denote the arrow from $x$ to $y$ for $i=1,2,3$. Define $\delta^{-}\left(a_{x, x}^{(i)}\right)=x \otimes a_{x, x}^{(i)}, \delta^{+}\left(a_{x, x}^{(i)}\right)=$ $a_{x, x}^{(i)} \otimes x, g \cdot a_{x, x}^{(i)}=a_{g x, g x}^{(i)}, a_{e, e}^{(i)} \cdot g=\chi_{e}^{(i)}(g) a_{x g, x g}^{(i)}$ for $x \in G, i=1,2,3$. By [4], $k Q_{1}$ is a $k G-$ Hopf bialgebra. Therefore, it follows from Theorem 7 that $\left(\left(k Q^{a}\right)^{\mathrm{cop}} \bowtie_{\tau} k Q^{c},\left\{R_{n}\right\}\right)$ is a local quasitriangular Hopf algebra and for every finite dimensional $\left(k Q^{a}\right)^{\text {cop }} \bowtie_{\tau} k Q^{c}$-module $M$, $C^{\left\{R_{n}\right\}}$ is a solution of Yang-Baxter equations on $M$.

By the way, we obtain the relation between path algebras and path coalgebras by Theorem 5 .

Corollary 1. Let $Q$ be a finite quiver over finite group $G$. Then Path algebra $k Q^{a}$ is algebra isomorphic to subalgebra $\sum_{n=0}^{\infty}\left(\square_{k G}^{n} k Q_{1}^{c}\right)^{*}$ of $\left(k Q^{c}\right)^{*}$.

Proof. Let $A=(k G)^{*}$ and $M=k Q_{1}^{c}$. It is clear that $\xi_{k Q_{1}^{c}}$ is a $k G$-bicomodule isomorphism from $k Q_{1}^{c}$ to $\left(k Q_{1}^{a}\right)^{*}$. See that

$$
T_{B^{*}}^{c}\left(M^{*}\right)=T_{(k G)^{* *}}^{c}\left(\left(k Q_{1}^{a}\right)^{*}\right) \stackrel{\nu_{1}}{\cong} T_{k G}^{c}\left(\left(k Q_{1}^{a}\right)^{*}\right) \stackrel{\nu_{2}}{\cong} T_{k G}^{c}\left(k Q_{1}^{c}\right)=k Q^{c},
$$

where $\nu_{1}=T_{k G}^{c}\left(\sigma_{k G}^{-1} \pi_{0}, \pi_{1}\right), \nu_{2}=T_{k G}^{c}\left(\pi_{0},\left(\xi_{k Q_{1}^{c}}\right)^{-1} \pi_{1}\right)$. Obviously, $\nu_{1}$ and $\nu_{2}$ are coalgebra isomorphism. Consequently, it follows from Theorem 5 that $k Q^{a}$ is algebra isomorphism to subalgebra $\sum_{n=0}^{\infty}\left(\square_{k G}^{n} k Q_{1}^{c}\right)^{*}$ of $\left(k Q^{c}\right)^{*}$.

\section{Acknowledgement}

The authors were financially supported by the Australian Research Council. S.Z. thanks Department of Mathematics, University of Queensland for hospitality.

\section{References}

[1] Auslander M., Reiten I., Smalø S.O., Representation theory of Artin algebras, Cambridge University Press, 1995.

[2] Belavin A.A., Drinfel'd V.G., solutions of the classical Yang-Baxter equations for simple Lie algebras, Functional Anal. Appl. 16 (1982), no. 3, 159-180.

[3] Chin W., Montgomery S., Basic coalgebras, in Modular Interfaces (1995, Riverside, CA), AMS/IP Stud. Adv. Math., Vol. 4, Amer. Math. Soc., Providence, RI, 1997, 41-47.

[4] Cibils C., Rosso M., Hopf quivers, J. Algebra 254 (2002), 241-251, math.QA/0009106.

[5] Cibils C., Rosso M., Algebres des chemins quantiques, Adv. Math. 125 (1997), 171-199.

[6] Drinfel'd V.G., Quantum groups, in Proceedings International Congress of Mathematicians (August 3-11, 1986, Berkeley, CA), Amer. Math. Soc., Providence, RI, 1987, 798-820.

[7] Dascalescu S., Nastasescu C., Raianu S., Hopf algebras: an introduction, Marcel Dekker Inc., 2001.

[8] Doi Y., Takeuchi M., Multiplication alteration by two-cocycles - the quantum version, Comm. Algebra 22 (1994), 5715-5732.

[9] Jantzen J.C., Lectures on quantum groups, Graduate Studies in Mathematics, Vol. 6, Amer. Math. Soc., Providence, RI, 1996.

[10] Majid S., Algebras and Hopf algebras in braided categories, in Advances in Hopf Algebras (1992, Chicago, IL), Lecture Notes in Pure and Appl. Math., Vol. 158, Marcel Dekker, New York, 1994, 55-105.

[11] Montgomery S., Hopf algebras and their actions on rings, CBMS Regional Conference Series in Mathematics, Vol. 82, Amer. Math. Soc., Providence, RI, 1993. 
[12] Nichols W., Bialgebras of type one, Comm. Algebra 6 (1978), 1521-1552.

[13] Lusztig G., Introduction to quantum groups, Progress in Mathematics, Vol. 110, Birkhäuser, Boston, MA, 1993.

[14] Kassel C., Quantum groups, Graduate Texts in Mathematics, Vol. 155, Springer-Verlag, New York, 1995.

[15] Sweedler M.E., Hopf algebras, Benjamin, New York, 1969.

[16] Yang C.N., Ge M.L. (Editors), Braid group, knot theory and statistical mechanics, World Scientific, Singapore, 1989.

[17] Zhang S., Chen H.-X., The double bicrossproducts in braided tensor categories, Comm. Algebra 29 (2001), 31-66. 